\title{
Efficacy and safety of nucleoside analogs on blocking father-to-infant vertical transmission of hepatitis B virus
}

\author{
LI-HAU CAO ${ }^{1}$, PEI-LI ZHAO ${ }^{1}$, ZHI-MIN LIU ${ }^{1}$, SHAO-CHUN SUN ${ }^{2}$, \\ DONG-BO XU ${ }^{1}$, JI-DONG ZHANG ${ }^{1}$ and MEI-HUA SHAO ${ }^{1}$ \\ ${ }^{1}$ Liver Disease Center, Qinhuangdao Third Hospital; ${ }^{2}$ Qinhuangdao Women's and Children's Hospital, \\ Qinhuangdao, Hebei 066000, P.R. China
}

Received June 25, 2014; Accepted February 18, 2015

DOI: $10.3892 / \mathrm{etm} .2015 .2379$

\begin{abstract}
The aim of the present study was to observe the efficacy and safety of nucleoside analogs in inhibiting father-to-infant vertical transmission of hepatitis B virus (HBV). Nucleoside analogs compete with HBV DNA polymerase substrate to inhibit DNA polymerase, thus preventing the replication of HBV DNA. A case group and control group were recruited for the study. Between March 2006 and March 2012 at the Liver Disease Center of Qinhuangdao Third Hospital, a total of 201 couples were recruited for the case group. In each case, the father tested positive the following HBV markers: Hepatitis B surface antigen (HBsAg), hepatitis B e antigen (HBeAg), antibodies against the hepatitis B core antigen (anti-HBc) and HBV DNA. In total, 189 male patients presented with abnormal liver function $(94.0 \% ; 189 / 201)$. Prior to pregnancy, all the males in the case group were required to test negative for HBV DNA and exhibit normal liver function, while the females were required to test positive for antibodies against HBsAg (anti-HBs). In total, 188 couples comprised the control group. The couples were recruited between March 2006 and March 2012 in the Prenatal Clinic of Qinhuangdao Women's and Children's Hospital. The fathers tested positive for $\mathrm{HBsAg}, \mathrm{HBeAg}$, anti-HBc and HBV DNA. With regard to the females, $\mathrm{HBsAg}$ tests were all negative and anti-HBs tests were positive. In the case group, there were no $\mathrm{HBsAg-positive} \mathrm{or} \mathrm{HBV}$ DNA-positive newborns, while anti-HBs tests were all positive; thus, the father-to-infant HBV vertical transmission was successfully inhibited. In the control group, 147/188 newborns tested positive for anti-HBs at birth, accounting for $78.2 \%$. In addition, 28 newborns were positive for HBV DNA (14.9\%), and 19 newborns tested positive for HBsAg (10.1\%).
\end{abstract}

Correspondence to: Dr Li-Hau Cao, Liver Disease Center, Qinhuangdao Third Hospital, 222 Jianguo Road, Qinhuangdao, Hebei 066000, P.R. China

E-mail: clh2777@163.com

Key words: nucleoside analogs, efficacy, safety, block, hepatitis B virus, father-to-infant vertical transmission
Statistically significant differences were observed between the two groups with regard to these parameters. However, no statistically significant differences in gestational age, birth weight, birth height, 1- and 8-min Apgar scores, presence of jaundice, other internal and surgical diseases, delivery mode and other birth information were observed when comparing the case group with the control group. Furthermore, there were no fetal malformations or stillbirths in the two groups. In the HBV DNA-positive fathers prior to pregnancy, antiretroviral therapy resulted in a reduced virus load. Therefore, blocking father-to-infant HBV vertical transmission maximally was important. The use of antiviral nucleoside analogs prior to pregnancy was shown to be safe. When the benefits outweighed the risks, the fathers who wanted to have a child continued to use antiviral therapy. However, the sample size of the present study was small, and an increased number of cases and longer follow-up times are required. In addition, the use of nucleoside analogs requires further in-depth assessment from the point of view of prenatal and postnatal care.

\section{Introduction}

The incidence of hepatitis B virus (HBV) infection is high in China. However, through the application of a hepatitis $\mathrm{B}$ vaccination nationwide, the transmission of $\mathrm{HBV}$ has gradually become controlled. In 2006, the National Hepatitis Epidemiological Survey revealed that the hepatitis B surface antigen ( $\mathrm{HBsAg}$ ) carrier rate in the general population aged between 1 and 59 years was $7.18 \%$, while the HBsAg carrier rate in children ages $<5$ years was only $0.96 \%(1,2)$. According to the investigation by Blumberg in 1977 (3), HBV DNA may be integrated into genes in host germ cells through a pedigree method, from which the virus can spread to offspring. An increasing number of studies have detected HBV DNA in semen and sperm (4). With the development of transgenic technology, certain studies $(5,6)$ have performed thorough research into the sperm pathway as a mode of transmission. In a previous study, the egg of a golden hamster was fertilized with the sperm of patients with $\mathrm{HBV}$; in the human sperm chromosome specimens, a virus probe hybridization signal was observed. During fertilization, the human sperm containing the integrated HBV DNA was 
not selectively eliminated, and the fertilization process was successfully completed. Thus, the uptake and detection of HBV DNA in sperm has been confirmed. The majority of scholars hypothesize that the motility of HBV-infected sperm is not affected, and that the sperm can successfully combine with the egg for further development (7). In 2006, Ali et al (8) demonstrated that the sperm-mediated HBV gene can be expressed in early embryonic cells, providing direct evidence that $\mathrm{HBV}$ can be passed to offspring from the parents. Father-to-child HBV transmission pathways can be divided into horizontal and vertical transmission pathways. With regard to horizontal transmission, the father carrying $\mathrm{HBV}$ is able to pass the virus through daily contact with the newborn since the neonatal immune system is weak. However, the process in vertical transmission is via germ cells. Horizontal transmission of HBV can be prevented by joint injections of hepatitis B immunoglobulin and hepatitis B vaccine (HBVac). However, vertical transmission occurs through germ cells. In particular, fathers positive for HBsAg, hepatitis B e antigen ( $\mathrm{HBeAg})$ and hepatitis $\mathrm{B}$ core antigen (anti-HBc) are more likely to transmit the infection to the infant via vertical transmission; father-to-child transmission is the second most important pathway of HBV vertical transmission (9). Methods of blocking father-to-infant vertical transmission remain limited in the medical field; thus, further research is required.

Immunological methods of blocking HBV vertical transmission are one of the main strategies in current clinical treatment; however, $20 \%$ of neonates exhibit immune failure, which is associated with HBV DNA levels in the serum of pregnant women (10). When the maternal serum level of HBV DNA is $>10^{8} \mathrm{IU} / \mathrm{ml}$, the intrauterine infection rate remains as high as $43 \%$, even with the application of immunological methods. However, when the HBV DNA serum level is $<10^{6} \mathrm{IU} / \mathrm{ml}$, there is a $30 \%$ reduction in the risk of mother-to-child HBV transmission (11). Efficient and safe anti-HBV drugs should be administered to pregnant women with a high viral load to inhibit HBV replication. With regard to increasing the inhibition rate of mother-to-infant HBV transmission, a previous study found that lamivudine was successful at inhibiting HBV intrauterine infection (12). Based on previous research investigating the inhibition of mother-to-infant HBV transmission, it has been hypothesized that nucleoside antiviral drugs may also be effective for block father-to-infant HBV transmission (10). Nucleoside analogs, which include lamivudine, adefovir, telbivudine and entecavir, compete with HBV DNA polymerase substrate to inhibit DNA polymerase, thus preventing the replication of HBV DNA and potentially mitigating chronic hepatitis B. Therefore, the aim of the present study was to observe the clinical efficacy and safety of HBV nucleoside analogs in inhibiting father-to-infant HBV transmission.

\section{Materials and methods}

Case group and control group selection. In the case group, outpatients were selected according to the order of hospital admission, between March 2006 and March 2012 in Qinhuangdao Third Hospital (Qinhuangdao, China). In total, 201 couples who were willing to undergo the study were selected. The patients were required to have good compli- ance, undergo regular consultations at the hospital and be willing to undergo follow-up examinations. During the consultations, the HBV markers (HBVM) of the fathers were assessed. For the case group, the fathers were required to test positive for $\mathrm{HBsAg}, \mathrm{HBeAg}$, antibodies against anti-HBc and HBV DNA. In total, 189 cases had abnormal liver function $(94.0 \%, 189 / 201)$. The exclusion criteria of HBVM of fathers were as follows: $\mathrm{HBsAg}$ positive, anti-HBe positive, anti-HBc-positive or $\mathrm{HBsAg}$-positive, anti-HBc positive, $\mathrm{HBV}$ DNA positive cases were not included in this group. The liver function of the mothers was normal and the HBVMs were as follows: Anti-HBs- or anti-HBs-positive, anti-HBc- or anti-HBs-positive, anti-HBe-positive and anti-HBc-positive. In total, 139 cases who had previously undergone immunization with the HBVac or had acquired immunity via a previous HBV infection were included; these patients accounted for $69.2 \%(139 / 201)$ of the case group. A total of 62 cases $(30.8 \%$, $62 / 201$ ) were anti-HBs-negative. For all the couples in the case group, prior to pregnancy, the fathers were required to be HBV DNA-negative and exhibit normal liver function, while the mothers were required to be anti-HBs-positive.

In the control group, 188 couples were selected from 1,957 couples who received an antenatal examination in Qinhuangdao Women's and Children's Hospital (Qinhuangdao, China) from between March 2006 and March 2012. In each father, liver function was normal and the following HBVMs were present: HBsAg-positive, $\mathrm{HBeAg}$-positive, anti-HBc-positive and HBV DNA-positive. In total, 13 cases had a quantitative viral load of $\leq 10^{6} \mathrm{IU} / \mathrm{ml}$, while 175 cases had a virus quantitative value of $>10^{6} \mathrm{IU} / \mathrm{ml}$. In the females, no HBVM detection was performed prior to the pregnancy, and each patient had no memory of receiving a HBVac injection. Liver function was normal and the HBVMs were as follows: Pregnant women were HBsAg-negative, anti-HBs-positive or anti-HBs-positive, anti-HBc-positive or anti-HBs-positive, anti-HBe-positive, anti-HBc positive. In total, 188 controls were included. HBVM were all negative, and only anti-HBc-positive pregnant women were excluded from this study.

All the selected couples met the following criteria: i) Negative testing for serum hepatitis A, C, D and E viruses and HIV, no alcoholic liver disease and autoimmune liver disease; ii) normal renal function; iii) provided written informed consent; iv) fathers had a history of HBV varying between 2 and 23 years; v) newborn venous blood samples were collected at birth. Umbilical cord blood was defined as residual blood in the placenta and umbilical cord, and the fetal umbilical cord was ligated to prevent mixing with contaminating factors in the mother's blood. Factors that may have potentially contaminated the umbilical cord blood included placenta previa, placental abruption and various other factors associated with caesarean delivery. This study was approved by the Ethical Committee of Qinhuangdao Third Hospital and informed consent was obtained from all couples.

Observed indicators in the case group. Prior to pregnancy, liver function, HBVMs and the HBV DNA load were assessed in the fathers before and after antiretroviral treatment, and at 1 , 3, 6,9 and 12 months after antiretroviral treatment. With regard 
Table I. Characteristics of the fathers in the two groups.

\begin{tabular}{|c|c|c|c|c|c|}
\hline Group & Cases (n) & Age (years) & Height $(\mathrm{cm})$ & Weight (kg) & $\begin{array}{l}\text { Antiviral treatment } \\
\text { before pregnancy }(n)\end{array}$ \\
\hline Case & 201 & $29 \pm 2.1$ & $176 \pm 5.1$ & $65 \pm 5.1$ & 201 \\
\hline Control & 188 & $28 \pm 1.9$ & $174 \pm 6.3$ & $66 \pm 5.5$ & 0 \\
\hline$\chi^{2}(t)$ & & $t=0.612$ & $\mathrm{t}=0.427$ & $\mathrm{t}=0.231$ & $\chi^{2}=389$ \\
\hline P-value & & 0.574 & 0.691 & 0.829 & $<0.001$ \\
\hline
\end{tabular}

Table II. Characteristics of the mothers in the two groups.

\begin{tabular}{|c|c|c|c|c|c|c|}
\hline Group & Cases (n) & Age (years) & Height $(\mathrm{cm})$ & Weight (kg) & Gravidity (n) & $\begin{array}{l}\text { Quantitative anti-HBs } \\
\text { before pregnancy (IU/l) }\end{array}$ \\
\hline Case & 201 & $25 \pm 2.3$ & $160 \pm 5.1$ & $55 \pm 5.1$ & $2 \pm 1.0$ & $497 \pm 35.7$ \\
\hline Control & 188 & $26 \pm 2.0$ & $158 \pm 5.7$ & $53 \pm 5.9$ & $2 \pm 1.0$ & $505 \pm 38.8$ \\
\hline t-value & & 0.85 & 0.453 & 0.444 & 0.00 & 0.263 \\
\hline P-value & & 0.443 & 0.674 & 0.680 & 1.00 & 0.806 \\
\hline
\end{tabular}

anti-HBs, antibody against hepatitis B surface antigen.

Table III. Common complications during pregnancy in the two groups.

\begin{tabular}{lcccc}
\hline Group & Cases & Hypertension & Pre-eclampsia & Diabetes \\
\hline Case (n) & 201 & 6 & 0 & 0 \\
Control (n) & 188 & 5 & 0 & 0 \\
$\chi^{2}$ & & 0.037 & & \\
P-value & & 0.874 & & \\
\hline
\end{tabular}

to the females, liver function and HBVMs were detected prior to pregnancy. In total, 62 cases tested negative for anti-HBs, which was detected prior to the HBVac and at 1 and 7 months after the HBVac.

Observed indicators in the control group. Liver function and HBVMs were detected in the mothers, while liver function, HBVMs and the HBV DNA load were detected in the fathers.

Detection methods. Venous blood samples from the infants were immediately collected after birth for assessment of the HBVMs and the HBV DNA viral load. HBVMs and the HBV DNA viral load were measured using the same sample from the newborns in the case and control groups. To assess the HBVMs, an electrochemiluminescence method was used with an automatic electrochemiluminescence immunoassay analyzer (cobas-e-411; Roche Diagnostics, Indianapolis, IN, USA); $\mathrm{HBV} \mathrm{HBsAg}$, anti-HBs, $\mathrm{HBeAg}$, anti-HBe and anti-HBc electrochemiluminescence detection kits were purchased from Roche Diagnostics. The quantitative assessment of HBV DNA was performed using quantitative polymerase chain reaction (PCR). PCR reagents, including HBV DNA quantitative fluorescence diagnostic kits, were provided by Hunan Shengxiang Biotechnology Co., Ltd. (Changsha, China). A quantitative PCR instrument (SLAN; Hunan Shengxiang Biotechnology Co., Ltd.) was used for detection, following the protocol described by Germer et al (13).

Treatment. Of the 201 couples in the case group, HBVM detection revealed that 62 mothers were anti-HBs-negative; thus, pre-pregnancy vaccination with HBVac was performed via three injections at 0,1 and 6 months. Primary vaccinees were administered $20 \mu \mathrm{g}$ of recombinant yeast, while previously vaccinated patients that did not produce anti-HBs received $40 \mu \mathrm{g}$, to ensure the presence of anti-HBs prior to pregnancy. A total of 139 mothers who tested positive for anti-HBs did not receive the HBVac injection, and all 201 mothers were shown to be anti-HBs positive prior to pregnancy. All the 201 fathers were carriers of HBV DNA and subsequently received antiviral nucleoside analog treatment. In total, 52 cases were administered lamivudine (100 mg, once per day), 24 cases received adefovir (10 $\mathrm{mg}$, once per day), 87 cases had telbivudine (600 mg, once per day) and 38 cases were administered entecavir ( $0.5 \mathrm{mg}$, once per day). Patients were administered different types of nucleoside analog according to their illness, drug price (certain patients lacked funding for particular treatments) and other factors.

In the 188 couples comprising the control group, all the mothers were anti-HBs positive, and no treatment was conducted during the pregnancy. In addition, the 188 fathers were HBV carriers, and also received no treatment prior to pregnancy.

Statistical analysis. SPSS 16.0 statistical software (SPSS, Inc., Chicago, IL, USA) was used for statistical analysis. Continuous data were compared using the $t$-test, while categorical data 
Table IV. General situation of the neonates at birth.

\begin{tabular}{|c|c|c|c|c|}
\hline Observed indicators & Case group $(n=201)$ & Control group $(\mathrm{n}=88)$ & $\chi^{2}(\mathrm{t})$ & P-values \\
\hline Pregnancy week & $39.21 \pm 1.21$ & $39.01 \pm 1.32$ & $\mathrm{t}=0.193$ & 0.856 \\
\hline Weight (kg) & $3.31 \pm 0.31$ & $3.32 \pm 0.32$ & $\mathrm{t}=0.039$ & 0.971 \\
\hline Height (cm) & $49.71 \pm 1.57$ & $49.62 \pm 1.51$ & $\mathrm{t}=0.072$ & 0.946 \\
\hline Gender, M/F (n) & 98/69 & $95 / 66$ & $\chi^{2}=0.004$ & 0.953 \\
\hline 1 min Apgar score & $9.85 \pm 0.51$ & $9.79 \pm 0.49$ & $\mathrm{t}=0.147$ & 0.890 \\
\hline 8 min Apgar score & $9.86 \pm 0.57$ & $9.83 \pm 0.58$ & $\mathrm{t}=0.064$ & 0.952 \\
\hline Jaundice (n) & 17 & 16 & $\chi^{2}=0.000$ & 0.985 \\
\hline Other internal and surgical diseases (n) & 0 & 0 & & \\
\hline Delivery mode, caesarean/head (n) & $104 / 97$ & $98 / 90$ & $\chi^{2}=0.006$ & 0.939 \\
\hline
\end{tabular}

M, male; F, female.

Table V. HBVM status of the neonates at birth in the two groups.

\begin{tabular}{lcccc}
\hline Group & Cases (n) & anti-HBs $(+)$ & HBsAg $(+)$ & HBV DNA (+) \\
\hline Case & 201 & 201 & 0 & 0 \\
Control & 188 & 147 & 19 & 28 \\
$\chi^{2}$ & & 138.278 & 21.357 & 32.258 \\
P-value & & $<0.001$ & $<0.001$ & $<0.001$ \\
\hline
\end{tabular}

HBVM, hepatitis B virus marker; anti-HBs, antibody against hepatitis B surface antigen; HBsAg, hepatitis B surface antigen.

were compared using the $\chi^{2}$ test. $\mathrm{P}<0.05$ was considered to indicate a statistically significant difference.

\section{Results}

Patient characteristics. Baseline characteristics of the couples in the two groups are shown in Tables I and II. The occurrence of complications during pregnancy in the two groups is shown in Table III. In addition, the general situation and HBVM status of the neonates at birth is shown in Table IV. In total, $167 / 201$ fathers in the case group were undergoing antiretroviral treatment at the point of conception, while 34 fathers had become HBV DNA-negative and withdrawn from the antiviral drugs between 3 and 6 months prior to conception.

HBVMs in the neonates of the two groups. Of the 167 patients in the case group who were undergoing medication at conception, 121 cases were determined to be HBV DNA-negative prior to conception, and no newborns were born as HBsAg-positive or HBV DNA-positive. In the remaining 46 cases, the fathers exhibited normal liver function; however, the patients not become HBV DNA-negative, with HBV DNA viral loads ranging between $1.1 \times 10^{3}$ and $8.9 \times 10^{4} \mathrm{IU} / \mathrm{ml}$. No neonate was born positive for $\mathrm{HBsAg}$. In the case group, no newborns tested positive for HBV DNA. In the control group, $147 / 188$ newborns were shown to have anti-HBs at birth, accounting for $78.2 \%$ (147/188). However, 28/188 newborns were HBV DNA-positive (14.9\%), and 19/188 newborns were HBsAg-positive (10.1\%).

\section{Discussion}

In 1985, Hadchouel et al (14) used molecular hybridization to find that integrated HBV DNA existed in the sperm of three patients with acute hepatitis $\mathrm{B}$, which demonstrated the existence of father-to-infant vertical transmission. Tajiri et al (15) also found evidence of father-to-child hepatitis transmission at a molecular biology level. From these studies, the possibility of father-to-child transmission of HBV was identified, and with further research, the existence of HBV father-to-child transmission was confirmed at the molecular level. The results by Huang et al (16) revealed that a HBV infection can cause sperm chromosome mutations. The integration between HBV DNA and the sperm chromosome was random and at multiple loci, which further increases the instability of the chromosomes, indicating that HBV infection may have a widespread impact by altering the genetic composition generation after generation. In 2004, Englert et al (17) reported that with assisted reproductive technology (ART), washed gradient centrifugation can be used to reduce the HBV load and avoid transmission to embryos. The sperm underwent washing and ART, and subsequently the spread of HBV was reduced. However, as a preventative method of father-to-infant vertical transmission, ART has a number of difficulties with regard to clinical implementation. Therefore, the identification of a convenient and effective method to inhibit father-to-infant vertical transmission is required.

Nucleoside analogs can act on the polymerase region of $\mathrm{HBV}$, and can substitute a similarly structured nuclei 
extending toward the desired nucleoside polymerase chain into the viral replication process (18). The chain extension is subsequently terminated to inhibit viral replication. Thus, this treatment method can be used to inhibit viral replication to a high degree. In recent years, nucleoside (acid) antiviral drugs have become increasingly used for the treatment of chronic hepatitis. However, since the duration of the drug treatment is very long, only a quarter of the patients are estimated to terminate the treatment within five years, and the majority of patients require long-term use. Therefore, a number of patients undergoing treatment are likely to experience fertility problems. In addition, interferon antiviral treatment may have an impact on sperm. Previous studies have reported that in male patients undergoing long-acting interferon and ribavirin therapy, the sperm count was reduced, the sperm appeared abnormal and the sperm DNA chromosome structure revealed certain abnormalities (19). In general, these effects gradually returned to normal within eight months after discontinuation; therefore, interferon therapy is known to markedly affect sperm. During the treatment, fertility was deemed to not be suitable. However, there has been no evidence indicating that the therapeutic dose of nucleoside analogs can cause sperm abnormalities.

Four nucleoside analogs have been specifically described with regard to reproductive toxicity and pregnancy medication: i) Lamivudine: Reproduction studies in animals indicated that the drug was not teratogenic, and had no effect on the male and female reproductive capacity; ii) adefovir: Animal studies demonstrated no effect on the fertility of male and female animals; iii) telbivudine: In vivo and in vitro studies did not reveal genotoxicity, and a clastogenic effect was not observed in the gene mutation analysis. Furthermore, in male and female rats, a dose 14 times the systemic exposure of the human therapeutic dose did not result in evidence of impaired fertility; iv) entecavir: In reproductive toxicity studies, the fertility in male and female rats was not affected. In toxicology studies, when the dose reached $\geq 35$ times the human dose, the degeneration of the vas deferens was observed in rodents and dogs. However, in monkey experiments, a change was not observed in the testis. In summary, the four types of drug were shown to be relatively safe in humans. Only with an drug overdose was toxicity and potential carcinogenicity unable to be excluded. The results of the present study revealed no statistically significant differences with regard to gestational age, birth weight, birth height, 1- and 8-min Apgar scores, jaundice, other internal and surgical diseases, mode of delivery and other birth data in the neonate when comparing the case and control groups. In addition, there were no fetal malformations or stillbirths in the two groups. Furthermore, the results indicated that neonatal infant HBV vertical transmission and the presence of HBsAg in the father exerted no statistically significant effects on the aforementioned indicators (Table IV). Previous studies have found that the rate of sperm chromosome aberrations in fathers infected with HBV was significantly higher compared with healthy controls, while the presence of HBV can also increase the rates of infertility, miscarriage, stillbirth, perinatal child mortality and the risk of fetal malformations (16,20-22); however, the results of the present study were different. The results demonstrated that the use of antiviral nucleoside analogs prior to pregnancy was safe in fathers. When the benefits outweighed the risks, the fathers who wanted to conceive a child were able to continue to use the antiviral therapy. However, the sample population size of the present study was small, and further studies with an increased number of cases and a longer follow-up time are required. In addition, further in-depth assessment from the point of view of prenatal and postnatal care is required.

From week 20 of pregnancy, the placenta exhibits an active $\mathrm{IgG}$ antibody transmission function from the mother to the fetus. Ayoda and Johnson (23) found that in 72 pregnant women with no HBV infection, who received the HBVac, 59\% of the neonates were HBsAb-positive at birth, indicating that fetal immunity was acquired successfully.

Results from previous studies $(7,9,24)$ have suggested that neonatal populations with a HBsAg-positive father have a higher prevalence of $\mathrm{HBV}$ infection; thus, they are a high risk population of HBV infection. However, the rate of transmission has differed among the various studies, and no consistent conclusions have been drawn $(25,26)$ One reason may be that the diagnostic criteria used by the institutes were not uniform. In addition, selected subjects of the study may differ. The results of the present study revealed that there were no newborns positive for HBsAg or HBV DNA in the case group, while all cases were positive for anti-HBs; thus, the father-to-infant HBV vertical transmission was successfully blocked. In the control group, 147/188 newborns were positive for anti-HBs at birth, accounting for $78.2 \%$. However, 28 newborns were positive for HBV DNA (14.9\%) and 19 newborns were HBsAg-positive $(10.1 \%)$. With regard to these factors, statistically significant differences were observed between the two groups (Table V).

In conclusion, the results of the present study demonstrated that when a father who is positive for HBV DNA undergoes antiretroviral therapy prior to pregnancy, negative turning occurs and the viral load decreases to a lower level, which is significant for maximally blocking father-to-infant HBV vertical transmission. However, due to the small number of samples, further in-depth research is required using an increased sample size. In addition, quantitative analyses of the HBV DNA viral load in fathers and the anti-HBs level in mothers is required in further stratified observations.

\section{References}

1. Liang X, Bi S, Yang W, Wang L, Cui G, Cui F, Zhang Y, Liu J Gong X, Chen Y, et al: Epidemiological serosurvey of hepatitis B in China-declining HBV prevalence due to hepatitis B vaccination. Vaccine 27: 6550-6557, 2009.

2. Liang X, Bi S, Yang W, Wang L, Cui G, Cui F, Zhang Y, Liu J, Gong X, Chen Y, Wang F, Zheng H, Wang F, Guo J, Jia Z, Ma J, Wang H, Luo H, Li L, Jin S, Hadler SC and Wang Y: Evaluation of the impact of hepatitis B vaccination among children born during 1992-2005 in China. J Infect Dis 200: 39-47, 2009.

3. Blumberg BS: Australia antigen and the biology of hepatitis B. Science 197: 17-25, 1977.

4. Zhang $M$ and Wang $H$ : Father infant vertical transmission of hepatitis B virus. Tianjin Yi Yao 34: 68-70, 2006 (In Chinese).

5. Huang JM, Huang TH, Qiu HY, Fang XW, Zhuang TG and Qiu JW: Studies on the integration of hepatitis B virus DNA sequence in human sperm chromosomes. Asian J Androl 4: 209-212, 2002.

6. Araki K, Miyazaki J, Hino O, et al: Expression and replication of hepatitis B virus genome in transgenic mice. Proc Natl Acad Sci USA 86: 207-211, 1989.

7. Ali BA, Huang TH and Xie QD: Detection and expression of hepatitis $\mathrm{B}$ virus $\mathrm{X}$ gene in one and two-cell embryos from golden hamster oocytes in vitro fertilized with human spermatozoa carrying HBV DNA. Mol Reprod Dev 70: 30-36, 2005. 
8. Ali BA, Huang TH, Salem HH and Xie QD: Expression of hepatitis B virus genes in early embryonic cells originated from hamster ova and human spermatozoa transfected with the complete viral genome. Asian J Androl 8: 273-279, 2006.

9. Komatsu H, Inui A, Sogo T, Hiejima E, Kudo N and Fujisawa T: Source of transmission in children with chronic hepatitis B infection after the implementation of a strategy for prevention in those at high risk. Hepatol Res 39: 569-576, 2009.

10. Han Z, Chen Z, Li L, Sun X, Sun Y, Zhao H and Su X: Lamivudine blocking efficacy and safety of vertical transmission of hepatitis B virus. Zhonghua Nei Ke Za Zhi 44: 378, 2005 (In Chinese)

11. Lin J, Liao S, Han JL, et al: Correlation between neonatal hepatitis B and maternal serum hepatitis B virus DNA copies. Pediatr Res 47: 342, 2000.

12. van Zonneveld M, van Nunen AB, Niesters HG, de Man RA, Schalm SW and Janssen HL: Lamivudine treatment during pregnancy to prevent perinatal transmission of hepatitis B virus infection. J Viral Hepat 10: 294-297, 2003.

13. Germer JJ, Qutub MO, Mandrekar JN, Mitchell PS and Yao JD: Quantification of hepatitis B Virus (HBV) DNA with a TaqMan HBV analyte-specific reagent following sample processing with the MagNA pure LC Instrument. J Clin Microbiol 44: 1490-1494, 2006.

14. Hadchouel M, Scotto J, Huret JL, Molinie C, Villa E, Degos F and Brechot C: Presence of HBV DNA in spermatozoa: A possible vertical transmission of HBV via the germ line. J Med Virol 16: 61-66, 1985

15. Tajiri H, Tanaka Y, Kagimoto S, Murakami J, Tokuhara D and Mizokami M: Molecular evidence of father-to-child transmission of hepatitis B virus. J Med Virol 79: 922-926, 2007.

16. Huang JM, Huang TH, Qiu HY, Fang XW, Zhuang TG Liu HX, Wang YH, Deng LZ and Qiu JW: Effects of hepatitis B virus infection on human sperm chromosomes. World J Gastroenterol 9: 736-740, 2003.
17. Englert Y, Lesage B, Van Vooren JP, Liesnard C, Place I, Vannin AS, Emiliani S and Delbaere A: Medically assisted reproduction in the presence of chronic viral diseases. Hum Reprod Update 10: 149-162, 2004.

18. Liu K and Xie L: Advances in the study of nucleoside antiviral drugs. Yao Xue Xue Bao 41: 689-693, 2006 (In Chinese).

19. Wang J, Song D, Zhang M, Zou H, Qian W and Peng N: Antiviral efficacy of different treatment of hepatitis B and its impact on sperm quality. Hai Nan Yi Xue Yuan Xue 24: 1732-1734, 2013 (In Chinese)

20. Livezey KW, Negorev D and Simon D: Increased chromosomal alterations and micronuclei formation in human hepatoma HepG2 cells transfected with the hepatitis B virus HBX gene. Mutat Res 505: 63-74, 2002.

21. Vicari E, Arcoria D, Di Mauro C, Noto R, Noto Z and La Vignera S: Sperm output in patients with primary infertility and hepatitis B or C virus; negative influence of HBV infection during concomitant varicocele. Minerva Med 97: 65-77, 2006

22. Ye F, Liu Y, Jin Y, et al: The effect of hepatitis B virus infected embryos on pregnancy outcome. Eur J Obstet Gynecol Reprod Biol 172: 10-14, 2014.

23. Ayoda EA and Johnson AO: Hepatitis B vaccine in pregnancy: immunogenecity, safety and transfer of antibodies to infants. Int J Gynaecol Obstet 25: 297-301, 1987.

24. Takegoshi $\mathrm{K}$ and Zhang W: Hepatitis B virus infections in families in which the mothers are negative but the fathers are positive for HBsAg. Hepatol Res 36: 75-77, 2006.

25. Wang S, Peng G, Li M, Xiao H, Jiang P, Zeng N and Wang Z: Identification of hepatitis B virus vertical transmission from father to fetus by direct sequencing. Southeast Asian J Trop Med Public Health 34: 106-113, 2003.

26. Cai QX and Zhu YY: Is hepatitis B virus transmitted via the male germ line? A seroepidemiological study in fetuses. Int J Infect Dis 17: e54-e58, 2013 